\title{
CINCO APUNTES SOBRE DERECHO Y POSTMODERNIDAD ${ }^{1}$
}

\author{
Andrés García Inda \\ Universidad de Zaragoza
}

A Luis Aranguren, Sebas Mora e Imanol Zubero, que interpretan los signos de este tiempo postmoderno, para transformarlo.

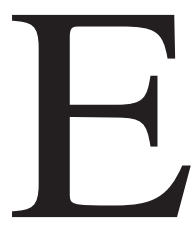

n las páginas que siguen, querríamos esbozar, muy brevemente, algunos de los rasgos, las tensiones y las perplejidades que caracterizan el llamado derecho de la postmodernidad ${ }^{2}$ y al que se enfrenta el jurista, el futuro profesional del derecho que sale de la Universidad. Y ello, como solemos insistir, no ya (o no tanto) porque la enseñanza del derecho deba simplemente «acomodarse» a ese discurso -seguramente del mismo modo que en ocasiones el discurso jurídico real no encajará con el derecho que se pueda enseñar en las Universidades-, sino al menos como forma de «saber» a qué juego se enfrenta el modelo que se enseña ${ }^{3}$ Y de entrada, habría que aludir en primer lugar al reconocimiento paradójico del valor que se atribuye al derecho en la sociedad actual: la tensión que subyace en el retorno y crisis -a la vez- de lo ju-

\footnotetext{
${ }^{1}$ Estas páginas fueron escritas durante el curso 1999-2000, como parte del Proyecto docente presentado en el primer ejercicio del concurso-oposición para la provisión de una plaza de profesor titular de filosofía del Derecho y Sociología Jurídica en la Universidad de Zaragoza.

${ }^{2}$ Cfr. A.-J. Arnaud y M J. Fariñas, Sistemas jurídicos: elementos para un análisis sociológico, Madrid, Universidad Carlos III / BOE, 1996, pp. 218-226 y 270-328; A. M. Hespanha, Panorama histórico da cultura jurídica europeia, Mem Martins, Publicaçoes Europa-Améri-

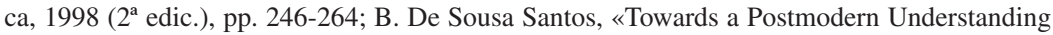
of Law», in Legal Culture and everyday life, Oñati Proceedings núm. 1, Oñati, IISJ, 1989, pp. 113-123; así como el monográfico de la Revista crítica de ciências sociais, 24 (1988); E. A. Russo, Teoría General del Derecho, Buenos Aires, Abeledo-Perrot, 1995, pp. 317-352. Véase otro abordaje diferente de la cuestión de la posmodernidad en J. Ballesteros, Postmodernidad: decadencia o resistencia, Madrid, Tecnos, 1989.

${ }^{3}$ Por poner un ejemplo: que el modelo de legitimidad piramidal haya sido desbordado en la práctica por las transformaciones acontecidas en el campo jurídico -empezando por todo lo que conlleva la inflación normativa y la legislación vía reglamento, o siguiendo por el reconocimiento del carácter creativo de la jurisprudencia- no quiere decir que ese modelo deba dejarse de lado, ni que haya que «aceptar» simplemente, y enseñar como «normal», el hecho de que en ocasiones, se acabe legislando a través de los reglamentos. Sobre el modelo de legitimidad piramidal vid. F. Ost, «Júpiter, Hércules, Hermes: tres modelos de juez» (trad de I. Lifante Vidal), Doxa 14 (1993), pp.
} 
rídico, entre una «desmedida fe en el derecho» $\mathrm{y}$ «un generalizado descreimiento» (desencanto y desencantamiento) respecto a las posibilidades del discurso jurídico ${ }^{4}$.

Por un lado, nos encontramos con una revalorización del derecho a la hora de comprender y justificar el poder ${ }^{5}$. Una vez despojado de todos sus atributos sagrados, el derecho vuelve a ser reconocido como a) un factor fundamental en la explicación de la vida social, y b) un elemento inevitable en su transformación. Por ejemplo, dice alguien ajeno al mundo jurídico:

«Cuando uno era un joven idealista y miraba con asombro y orgullo la Revolución de los Claveles, pensaba no sólo que había que cambiar el mundo, sino también que, para hacerlo, bastaba con la voluntad de eliminar lo viejo, con la mera fuerza de las ideas a flor de piel. Pasado el tiempo y curadas todas las gripes izquierdistas, pero, afortunadamente, sin matar el virus utópico que las provocaba, uno se arrepiente de no haber aprendido algo sobre dos de las cuestiones que más, me parece, podían servir para que los ideales tomen cuerpo en la realidad social. Me refiero al Derecho y a la Economía.

Hemos despreciado el Derecho porque nos parecía que las leyes, los reglamentos y los tribunales eran corsés para la espontaneidad de la vida; los abogados, males necesarios que viven de las desgracias ajenas («pleitos tengas») y para qué hablar de los notarios... En fin, que hemos ignorado cómo sólo aquello que se plasma en la letra, pequeña o grande, tiene posibilidades de ser argumentado, que los grandes enunciados no son operativos si no se concretan por escrito en el BOE, que un convenio colectivo no sirve de nada si no está bien formulado.» ${ }^{6}$

Esa «reconciliación» con el mundo jurídico no implica que haya que hacer del Derecho, nuevamente, el sagrado totem, el dios posible de una sociedad secularizada, ni que sea garantía de cambio o transformación. De hecho, en muchos casos, en ese retorno el discurso jurídico ha acabado por convertirse en la ancilla de la nueva teología del milenio: la Economía. Y en otros, no está exenta de matices ${ }^{7}$. Pero lo paradójico es que ese «retorno»

\footnotetext{
${ }^{4}$ N. M M $^{\text {a }}$ pózez Calera, Filosofía del Derecho, Granada, Comares, 1992, p. 14.

${ }^{5}$ L. Ferry y A. Renaut, 68-86. Itinéraires de l'individu, Paris, Gallimard, 1987, pp. 73-74; P. Bouretz, «La force du droit», en La force du droit. Panorama des débats contemporains, Paris, Éditions Esprit, 1991, pp. 9-11; A. García Inda, La violencia de las formas jurídicas, Barcelona, Cedecs, 1998, pp. 232-233.

${ }^{6}$ J. L. Sánchez Noriega, «Asignaturas pendientes», Noticias obreras 1128 (1994), p. 40 (592). También P. Flores D’Arcais, «Izquierda y legalidad», Jueces para la democracia 19/2 (1993), pp. 27-37 (esp. pp. 27-28), y más recientemente en El individuo libertario, trad. de J. Jordá, Barcelona, Seix Barral, 2001.

${ }^{7}$ Por ejemplo, en lo que hace referencia a la «sustitución» de la política por el derecho, sobre la que se debate a propósito de cuestiones como el papel de la jurisprudencia constitucional (vid. al respecto el número monográfico de la revista Le débat 64/1991), la revisión judicial de los derechos, la utilización del discurso jurídico con fines de política social, etc. y que alude a aquellos casos en los que el Derecho no sólo marca los límites o los márgenes de la
} 
o esa -matizada- «reconciliación» coexiste con lo que algunos entienden es una crisis de la capacidad regulativa del derecho (por la complejidad de las sociedades contemporáneas) o, cuando menos una crisis de la razón jurídica, una «pérdida de confianza en esa artificial reason que es la razón jurídica moderna, que erigió el singular y extraordinario paradigma teórico que es el Estado de derecho» ${ }^{8}$. O tal vez, cabría plantear la hipótesis, ese retorno y esa crisis se producen y alimentan mutuamente. Es la paradoja del amor/odio del censor, que André-Jean Arnaud plasma en la marginación y el retorno de la figura del juez a un mismo tiempo9 (y que sociológicamente podríamos relacionar con la persistente desconfianza en la Administración de Justicia, de los jueces y del derecho en general, con la reivindicación de su papel, y el protagonismo que llegan a cobrar en ocasiones). Es decir, se trata de una doble crisis de lo jurídico, arrastrada a lo largo de toda la segunda mitad del siglo XX: institucional, por un lado, dada la progresiva falta de eficacia del derecho y la aparición de zonas tanto de «a-legalidad» como de «i-legalidad»; y de legitimidad, por otro, por la pérdida de capacidad simbólica del discurso jurídico para representarse -léase: para ser reconocido- como el medio idóneo y justo de organización social ${ }^{10}$.

Ferrajoli distingue esquemáticamente tres aspectos de esa crisis de lo jurídico ${ }^{11}$. En primer lugar, dice, se trata de una «crisis de la legalidad, es de-

discusión política, sino que sustituye a ésta. Si bien existen aspectos positivos de esa sustitución (como la búsqueda de limitación del poder) no esta exenta de riesgos (fragmentación, confusión de esferas, «neutralización» del discurso político, etc.). Valga decir, en suma, que de ese proceso resulta una «contaminación» mutua: no sólo se formaliza o «juridifica» el discurso político; también se desformaliza o «politiza» el discurso jurídico.

${ }^{8}$ L. Ferrajoli, Derechos y garantías. La ley del más débil, trad. de P. Andrés Ibáñez y A. Greppi e introd. de P. Andrés Ibáñez, Madrid, Trotta, 1999, p. 18. Sobre el Estado de derecho, cfr. E. Díaz, Estado de derecho y sociedad democrática, Madrid, Cuadernos para el diálogo, 1979; R. de Asís, Una aproximación a los modelos de Estado de derecho, Madrid, Dykinson, 1999. Con todo, si realmente es verdad que se ha devaluado o ausentado esa razón jurídica habrá necesariamente que reinventarla: la cuestión es qué tipo de «nuevo sentido común jurídico» (por utilizar palabras de B. de Sousa Santos) se requiere hoy día. O el «equilibrio mental» necesario, como dice Arnaud, para llegar a hacerlo: «hay que resignarse a terminar constatando la ausencia de una razón jurídica que gobierne el derecho contemporáneo; algo que explica en parte el desconcierto de los juristas contemporáneos. En otros términos, debemos reconocer, nosotros, juristas diferentes, que no hemos encontrado todavía en el Derecho contemporáneo una razón que no sea ni orden ni paz. A nosotros nos corresponde inventarla», A.-J. Arnaud, «Los juristas frente a la sociedad (1975-1993)» (trad. de I. Lifante y V. Roca), Doxa 15-16 (1994), p. 1012.

${ }^{9}$ Cfr. A.-J. Arnaud, «Los juristas frente a la sociedad (1975-1993)», op. cit., p. 1002.

${ }^{10}$ Véase, A. M. Hespanha, Panorama histórico da cultura jurídica europeia, op.cit., pp. 230-231.

${ }^{11}$ Las transformaciones y crisis del método jurídico no son sino el reverso de la crisis de los presupuestos básicos sobre los que se levantó el edificio del derecho y la teoría liberal de 
cir, del valor vinculante asociado a las reglas por los titulares de los poderes públicos», y que se trasluce en ejemplos como la ineficacia del control y la ilegalidad del poder, la corrupción, el desplazamiento del Estado a sedes extra-legales, etc. En segundo lugar, «la inadecuación estructural de las formas del Estado de derecho a las funciones del Welfare State, agravada por la acentuación de su carácter selectivo y desigual de la crisis del Estado social», lo que se manifiesta en la «inflación legislativa provocada por la presión de los intereses sectoriales y corporativos, la pérdida de generalidad y abstracción de la leyes, la creciente producción de leyes-acto, el proceso de descodificación y el desarrollo de una legislación fragmentaria, incluso en materia penal, habitualmente bajo el signo de la emergencia y la excepción». Y en tercer lugar «la crisis del Estado nacional, y que se manifiesta en el cambio de los lugares de la soberanía, en la alteración del sistema de fuentes y, por consiguiente, en un debilitamiento del constitucionalismo». ${ }^{12}$

Es particularmente importante, en nuestro tiempo, la reflexión que pueda hacerse sobre esa crisis de la capacidad regulativa del Derecho en relación con las cuestiones relativas a los problemas identitarios, que ocupan un lugar cada vez más determinante en los conflictos de nuestro tiempo. Para algunos, precisamente, la incapacidad del Derecho para crear un régimen de convivencia viene dada por su inadecuación para proporcionar o para devolver a las colectividades el sentido de permanecer unidas. ${ }^{13}$

la época contemporánea, siglos XIX y XX: la democracia representativa y el principio de la legalidad (sometido a fuertes embates tanto en lo que respecta a la ley como fuente principal del derecho como a los procesos políticos de producción de la ley), la idea de Estado-nación y el positivismo. Véase sobre esos presupuestos: A. M. Hespanha, Panorama histórico da cultura jurídica europeia, op.cit., pp. 169-176.

12 L. Ferrajoli, Derechos y garantías, op. cit., pp. 15-17. Esa crisis estatal y de legalidad conduce a lecturas en términos de anomia (véase R. Dahrendorf, Ley y orden, trad. de L. M ${ }^{\mathrm{a}}$ Díez Picazo, Madrid, Civitas, 1994), o de entropía jurídica (J. L. Villar Palasí y J. L. Villar Ezcurra, «El derecho a la certidumbre jurídica y la incertidumbre de nuestro derecho», en Varios, La protección jurídica del ciudadano. Estudios en homenaje al prof. Jesús González Pérez (Tomo I), Madrid, Civitas, 1993, pp. 79-104). Y en relación con la opacidad y la no comprensión del discurso jurídico, C. M ${ }^{a}$ Cárcova, La opacidad del derecho, presentación de J. R. Capella, Madrid, Trotta, 1998. Sobre la crisis de la legalidad véase además L. Prieto Sanchís, Ley, principios, derechos, Madrid, Dykinson / Instituto de Derechos Humanos «Bartolomé de Las Casas», 1998; y L. Hierro, Estado de derecho. Problemas actuales, México, Fontamara, 1998, pp. 17-44.

${ }^{13} \mathrm{Ph}$. Delmas, El brillante provenir de la guerra, trad. de Pierre Jacomet, Santiago de Chile, Editorial Andrés Bello, 1996, pp. 22-23. Partiendo de la diferencia entre el orden (el sentimiento de estar juntos) y la organización (la convención de intereses), Philippe Delmas señala cómo en el ámbito internacional -y en parte sus tesis son aplicables a otros ámbitos más reducidos- el Derecho no sirve como instrumento para garantizar el orden, para potenciar y cuidar ese sentido de vida en común. Tras esa idea subyace una manifestación de la crisis del Derecho como instrumento de la política así como la reducción de ésta a mera técnica de gestión «organizativa». 
A poco que profundicemos, podremos darnos cuenta de que todas esas manifestaciones de la crisis tienen que ver con la globalización y el proceso de difuminación de las fronteras -reales y simbólicas- que mantenían las dicotomías propias del proyecto de la modernidad: Estado/sociedad civil, público/privado, formal/informal, jurídico/ajurídico... ${ }^{14}$ Esa difuminación, según algunos, obliga a la necesidad de nuevas formas de aproximación a la realidad, nuevos equilibrios mentales, un «nuevo sentido común», también en el caso de los juristas, que permita afrontar los retos, las tensiones -distinguir la «decadencia» de la «resistencia», por utilizar los términos del subtítulo del libro de J. Ballesteros; o la «emancipación» de la «dominación», tal como lo plantea B. De Sousa santos- propias del derecho de la postmodernidad. Esas tensiones, además, se convierten a su vez en objetivos, y vienen a constituir algunos de los criterios útiles para la definición cuando menos del talante posible en la formación del jurista. Algunas de ellas podrían ser las siguientes: la multiplicidad, la flexibilidad, la levedad, la rapidez y la paradoja. ${ }^{15}$

\section{Multiplicidad}

«El objeto denominado Derecho - dice Arnaud- se multiplica», y no sólo por la aparición de nuevos campos de estudio y de práctica, o de nuevas formas de relación, sino por «la extensión de la regulación social por la producción de normas (jurídicas) no gubernamentales» ${ }^{16}$. Se habla así de multiplicidad de actores jurídicos y de niveles de poder, de «multitud de miniracionalidades en el seno de una racionalidad global incontrolable», o de

${ }^{14}$ Sobre la polarización dicotómica y la ausencia de mediaciones como las dos características del proyecto de la modernidad, cfr. B. de Sousa Santos, «O Estado e o Direito na Transição Pós-Moderna: para um Novo Senso Comum sobre o Poder e o Direito», Revista Crítica de Ciências Sociais 30 (1990), pp. 13-16. Sobre la nueva función del Derecho en el proceso de globalización, cfr. B. de Sousa Santos, La globalización del derecho, Santafé de Bogotá, Universidad nacional de Colombia / ILSA, 1998; también los respectivos monográficos de las revistas Droit et Société 35 (1997), coordinado por W. de Lemos Capeller, y Anales de la Cátedra Francisco Suárez 32 (1995), sobre «mundialización económica y crisis político jurídica»; así como el volumen colectivo sobre Transformaciones del derecho en la mundialización, coordinado por J. R. Capella, Madrid, Escuela de Derecho Judicial, Consejo General del Poder Judicial, 1999; más reciente es el trabajo de J. E. Faria, El derecho ante la globalización económica, Madrid, Trotta, 2001. En nuestra cultura jurídica, además, muchas de las transformaciones que están en el origen de esas «crisis» tienen que ver con los cambios introducidos por el proceso de unificación y convergencia europea. A ese respecto, puede resultar sugerente el libro de A.-J. Arnaud, Pour une pensée juridique européenne, Paris, PUF, 1991.

${ }^{15}$ Nos ayudamos también, para este punto, de las propuestas culturales de I. Calvino, Seis propuestas para el próximo milenio, trad. de A. Bernárdez, Madrid, Siruela, 1990.

${ }^{16}$ A.-J. Arnaud, «Los juristas frente a la sociedad (1975-1993)», op. cit., p. 1004. 
una pluralidad de campos sociojurídicos ${ }^{17}$. En «la edad de la descodificación» ${ }^{18}$, la imagen del código como referente de un Derecho Uno, único e indivisible se ve así desbordada por la existencia no sólo de un Derecho plural, sino también de una pluralidad de derechos. El Derecho como metarrelato se disuelve en una pluralidad de pequeños relatos (decisiones, normas fragmentarias, experiencias jurídicas diversas, ordenamientos múltiples...), que apuntan a una nueva perspectiva, que entiende la vida cotidiana como un mundo de producción de normas, y analiza el propio saber jurídico desde el punto de vista de lo cotidiano ${ }^{19}$.

La conciencia de pluralidad que de ello se deriva -y que debe guiar la formación del jurista- no está exenta de tensiones, sino al contrario: esa conciencia y ese reconocimiento no supone la eliminación de lo singular, de lo diferente, porque la multiplicidad no tiene nada que ver con la generalidad, con el suministro de generalidades. «El seguimiento de la multiplicidad implica profundización en la singularidad (...): implica que, aún tomando como objeto de docencia o de investigación un concepto muy concreto, incluso algo nimio, uno ha de ser capaz de lograr que propicie el establecimiento de relaciones entre discursos jurídicos, niveles institucionales e, incluso, modelos de análisis distintos. Hasta la mayor trivialidad jurídica debería poder convertirse en un elemento de análisis potencialmente interminable» ${ }^{20}$. La multiplicidad es sinónimo así de la complejidad de una realidad jurídica plural e interdependiente; de un derecho que no se crea unidireccionalmente, que no sólo fluye de arriba abajo, sino también de abajo arriba, con los problemas de control que eso conlleva ${ }^{21}$.

La percepción de la pluralidad pone en cuestión la conciencia de la unidad (e incluso de la especie). Acostumbrados al monismo del Código, la

17 B. de Sousa Santos, La globalización del derecho, op. cit.; F. Ost, «Júpiter, Hércules, Hermes», op. cit., pp. 182-185. Las «minirracionalidades» no son necesariamente «racionalidades mínimas». Cfr. B. De Sousa Santos, De la mano de Alicia. Lo social y lo político en la postmodernidad, trad. de C. Bernal y M. García Villegas, Santafé de Bogotá, Siglo del Hombre Editores, 1998, p. 130.

18 Por utilizar la expresión que da título al libro de N. Irti, La edad de la descodificación, trad. e introd. de L. Rojo, presentación de A. Luna Serrano, Barcelona, Bosch, 1984.

${ }^{19}$ A. M. Hespanha, Panorama histórico da cultura jurídica europeia, op. cit., pp. 249-252. Y sobre el derecho de la vida cotidiana: A. Sarat \& Th. R. Kearns, Law in everyday life, Ann Arbor, Univ. Michigan Press («The Amherst Series in Law, Jurisprudence and Social Thought»), 1993.

${ }^{20}$ A. Serrano, «La rata en el laboratorio o la historia como observatorio jurídico», Anuario de Historia del Derecho Español LXII (1992), pp. 711-712.

${ }^{21}$ P. Casanovas, «Las formas sociales del derecho contemporáneo: el nuevo ius commune», Working Papers nº 146, Barcelona, Institut de Ciències Politiques i Socials, 1998, pp. 19-24. Pompeu Casanovas alude al cambio de la forma del derecho que se produce «de una forma 'normativa' a una forma común 'participativa'», a un «modelo organizativo» de derecho (p. 9). 
multiplicidad del «banco de datos» sorprende y desconcierta al jurista, y ese desconcierto inicial llega a convertirse en perplejidad y escepticismo, la respuesta de cuando no se tienen respuestas ${ }^{22}$. Para algunos, ese estado de perplejidad constituye el sumum intelectual ante la crisis del pensamiento racional. Sin embargo, el reto estriba precisamente en lo contrario, en abordar esa realidad múltiple sin caer en la generalidad, en desarrollar una conciencia de pluralidad sin instalarse en la perplejidad, en abordar el análisis de la diferencia y la singularidad sin apostar por el escepticismo. Se trata de superar aquellos escollos de los que no puede dar cuenta el pensamiento racional tradicional sin renunciar a él, y sin quedar hundidos en el «camino de la duda eterna: en el pantano de la separación entre el postodo y la prenada». La recuperación de la memoria a través de la historiografía, o el recurso a formas narrativas de aproximación a la realidad, por ejemplo, pueden sin duda contribuir a ese esfuerzo ${ }^{23}$.

Uno de los grandes riesgos que acompaña ese proceso es que la multiplicidad del fenómeno jurídico contribuya a aumentar la dispersión, uno de los grandes enemigos en la actividad del jurista. La existencia de enormes cantidades de información así como de múltiples vías para acceder a esa información es quizás la manifestación principal de ese riesgo, que en lo que respecta a la enseñanza y la formación de los profesionales del derecho, cobra especial importancia. Esa enseñanza, que corre el peligro de orientarse únicamente a proporcionar grandes cantidades de información, debe precisamente dedicarse a intentar que el jurista desarrolle las capacidades necesarias para «dominar» esos caudales. No se trata sólo de estar bien informado, sino de saber qué hacer con esa información; estar bien informado no es lo único importante, también es necesario pensar. ${ }^{24}$

22 «El mayor signo de desconcierto puede ser el no saber dónde situar al propio desconcierto». A.-J. Arnaud, «Los juristas frente a la sociedad (1975-1993)», op. cit., p. 1010.

23 Tomamos como ejemplo el abordaje que del choque cultural hace F. Fernández Buey, La gran perturbación. Discurso del indio metropolitano, Barcelona, Destino, 1995. También, por ejemplo, el desarrollo de seminarios sobre «Literatura y Derecho» en el marco de los programas de Teoría del Derecho tiene que ver con el intento de aproximar al jurista a unas realidades que, directamente o a través de otros caminos, permanecen inaccesibles o incomprensibles para él. Como dice Martha Nussbaum, la imaginación literaria (la «fantasía) es un ingrediente esencial de la postura ética por cuanto nos aproxima a realidades distantes o inaccesibles. M. Nussbaum, Justicia poética, trad. de C. Gardini, Barcelona, Editorial Andrés Bello, 1995. Esa posibilidad que ofrece la literatura de «vivir otras vidas» (al decir del escritor J. Jiménez Lozano) o de «reconocerse en lo desconocido» (como lo plantea el poeta A. Guinda) no consiste en reemplazar el razonamiento por las emociones, sino en incorporar la imaginación al razonamiento.

24 «Con un solo libro se puede formar un buen jurista, basta que el interesado lo lea con detenimiento y que tenga alguien cerca con quien quiera comentarlo; con los miles de libros $-\mathrm{y}$ de leyes y de sentencias- que hoy nos rodean y nos agobian es, en cambio, imposible». A. Nieto y T.-R. Fernández, El derecho y el revés. Diálogo epistolar sobre leyes, abogados y jueces, Barcelona, Ariel, 1998, p. 205. Es la dialéctica entre la cantidad y la calidad de la información. 


\section{Flexibilidad}

La flexibilidad -esto es: la posibilidad de cambiar las reglas sin destruir la organización- es quizás la característica más sobresaliente de la red como paradigma de la tecnología de la información; la red, dice M. Castells, es una «estructura abierta y flexible», capaz de expandirse sin límites ${ }^{25}$. Como hemos apuntado en otro lugar, además de la «extensión» del derecho se produce su «estiramiento» ${ }^{26}$, convirtiéndolo en un discurso dúctil, blando, flexible, por seguir utilizando la afortunada expresión de Jean Carbonnier ${ }^{27}$. Éste, Carbonnier, apuntaba a la necesidad de sobrepasar la rigidez del normativismo abstracto del legalismo y del conceptualismo. Sin embargo, como señala António Hespanha, hoy día la idea de derecho flexible tiene además otra genealogía intelectual: Por un lado, la atención a la identidad particular, a la individualidad y a la diferencia; por otro, el impacto que viene teniendo en la teoría de las organizaciones la tendencia a sustituir la estructura burocrática por una organización más flexible y maleable ${ }^{28}$. Como explica y sintetiza el historiador portugués:

«Ora, o direito tradicional corresponde, quase ponto por ponto, às tecnologias organizacionais das sociedades de «segunda vaga». É produto de una organização hiper-hierarquizada -o estado. Exprime-se em comandos de natureza genérica e abstracta, sejam eles as normas legais ou as proposições dogmáticas. Procura construir una versão das situações purificada de todos os conteúdos emocionais, reduzida apenas aos seus elementos racionais. E, finalmente, desenvolve técnicas de resolução de conflitos baseadas na intervenção, neutra e distnate, de um terceiro que, na avaliação das situações, procura manter-se emocionalmente não envolvido.

Em contrapartida, um direito para o novo tipo de organização teria que prescindir da generalidade e da abstracção. Teria que ser produzido «localmente», nas periferias que estão mais em contacto com as situações a regular. Mas teria ainda que prescindir de características proventura mais profundas do nosso

\footnotetext{
${ }^{25}$ M. Castells, La era de la información. Economía, sociedad y cultura. Vol. 1. La sociedad red, trad. de C. Martínez Gimeno, Madrid, Alianza, 1999, pp. 87-92 y 506-507. Sobre la rigidez y flexibilidad de los modelos jurídicos, véase V. Ferrari, Funciones del derecho, trad. de M J J. Añón y J. De Lucas, Madrid, Debate, 1989, pp. 150-155.

${ }^{26}$ A. García Inda y R. Susín Betrán, «Políticas sociales y Derecho», en Varios, Derecho y sociedad, Valencia, Tirant lo blanch, 1998, p. 150.

27 J. Carbonnier, Derecho flexible. Para una sociología no rigurosa del Derecho, trad. y prólogo de L. Díez-Picazo, Madrid, Tecnos, 1974. Otros, como G. Zagrebelsky, se refieren a El Derecho dúctil (trad. de M. Gascón y epílogo de G. Peces-Barba, Madrid, Trotta, 1995). Esa ductilidad está en consonancia con la reivindicación del protagonismo judicial, el recurso a los principios, etc. Véase al respecto L. Prieto Sanchís, Ley, principios, derechos, op. cit; Id., Sobre principios y normas. Problemas del razonamiento jurídico, Madrid, CEC, 1992.

${ }^{28}$ A. M. Hespanha, Panorama histórico da cultura jurídica europeia, op. cit., pp. 253-254.
} 
mundo jurídico, como o modelo da justiça adjudicatória (i. e., garantida por um «terceiro» independente e neutro), fomentando formas de composição pactada. Na verdade, a justiça «neutra» não considera uma parte muito importante das situações, o plano da emoção e da afectividade. Com isto, perde muita informação que seria indispensável para uma composição mais eficaz.» ${ }^{29}$

La demanda de flexibilización ${ }^{30}$ se traduce en un frecuente recurso a conceptos jurídicos indeterminados y estereotipos, la utilización de fórmulas pretendida o no pretendidamente ambiguas, la creación de amplias zonas de discrecionalidad «técnica», el aumento de los espacios de decisión no propiamente jurídicos dentro del derecho, la difuminación de las fronteras entre ámbitos jurídico y no jurídicos, etc.; todos ellos son algunos de los factores que han contribuido a esa transformación ${ }^{31}$. Más aún, según algunos la multiplicidad de lo jurídico hace insuficiente esa referencia a la flexibilidad: «Es a un Derecho «líquido», intersticial e informal al que estamos confrontados ahora. Un Derecho que, sin cesar de ser él mismo, se presenta en ciertas ocasiones en el estado fluido que le permite colocarse en las situaciones más diversas y ocupar así suavemente todo el espacio disponible, soportando -llegado el caso- fuertes compresiones» ${ }^{32}$.

¿Significa eso que desaparece de lo jurídico la idea de rigidez, del Derecho como «regla de acero», que da seguridad? Arnaud habla de fragilidad, de levedad de lo jurídico, e incluso recurre al mito del talón de Aquiles para subrayar el carácter delicado, incierto, precario, de «un Derecho frágil»; Ost en cambio prefiere pensar en la imagen de un metal flexible, como el mercurio, una regla líquida que le permite amoldarse a las sinuosidades de la piedra $^{33}$. En todo caso supone que el valor de la seguridad que el Derecho proporciona -la calculabilidad o previsibilidad propia del Derecho racional weberiano- también se transforma. Y que el jurista -y la formación del ju-

${ }^{29}$ Ibídem, p. 255.

${ }^{30}$ Véase al respecto, por ejemplo, L. Parejo Alfonso, Crisis y renovación en el derecho público, Madrid, CEC, 1991, p. 48, que alude precisamente a «las demandas de flexibilización de la programación administrativa».

${ }^{31}$ En derecho internacional, por ejemplo, se habla expresamente de derecho blando o fluido, de soft law, en el contexto de la protección del medio ambiente, los derechos humanos o el desarrollo económico internacional. Véase al respecto, por ejemplo, O. Elias y Ch. Lim, « «eneral Principles of Law», «Soft Law» and the identification of international law», Netherlands Yearbook of International Law, XXVIII (1997), pp. 3-49, especialmente pp. 45-48 (y la bibliografía allí citada). En lo que repecta a la cuestión de los conceptos jurídicos indeterminados, véase J. Almoguera Carreres, «Conceptos jurídicos indeterminados y jurisprudencia constitucional en el Estado social», en E. Olivas (edtr.), Problemas de legitimación en el Estado social, Madrid, Trotta, 1991, pp. 107-136.

${ }^{32}$ F. Ost, «Júpiter, Hércules, Hermes», op. cit., p. 187.

33 Ibídem, p. 187; A.-J. Arnaud, «Los juristas frente a la sociedad (1975-1993)», op. cit., pp. 1000 y 1004. 
rista- deberá proveerse de los medios técnicos y retóricos necesarios -habilidades, aptitudes, conocimientos- que le permitan trabajar con (o en) ese objeto jurídico flexible.

\section{Levedad}

Esa flexibilidad, según unos, o blandura, según otros, de lo jurídico, tiene que ver además con la «precariedad» del consenso que le sirve de fundamentación. El Derecho que, separado finalmente de la moral -siendo a su vez esta distancia la única forma posible de enjuiciarlo moralmente- busca en su propia dinámica la fuente de su fundamentación, realiza un esfuerzo parecido al del barón de Munchhausen, que consiguió salir de apuros -junto a su caballo- tirándose a sí mismo hacia arriba de su propia melena. Esa imagen ha servido a algunos para referirse las aporías del discurso ético en la actualidad ${ }^{34}$, y que afectan por lo tanto a la propia teoría de la justicia, cuya salida ante la crisis parece ser la de la ironía ${ }^{35}$. Tal vez esa supuesta crisis de la certeza sobre la idea de justicia encontró su formulación simbólica más palpable el día en que la fórmula presuntamente más antigua -y en todo caso la más utilizada en la historia- para referirse al problema de lo justo -«dar a cada cual lo que le corresponde»- fuera colocada como inscripción en el portal del campo de concentración nazi de Buchenwald.

Despojado así del peso de aquellas concepciones de lo justo que implicaban la realización de cualquier idea del bien (que puede ser cuestionada), el Derecho se concentra ahora en la consecución de lo correcto, o se refugia en una microética que impide pensar en problemas globales ${ }^{36}$. Sin embargo, como otros han puesto de relieve, la «neutralidad» que algunos predican del Estado y el Derecho no es sino el reverso de una determinada concepción cultural y social, la «debilidad axiológica»-expresión tal vez de la hegemonía de un pensamiento así considerado- suele traducirse en ocasiones en mera fuerza, y el «relativismo cultural» en que parece fundamentarse la neutralidad acaba por servir a la universalidad fáctica, consecuencia de la globalización ${ }^{37}$. El asunto afecta especialmente a la aproximación que podamos hacer a los derechos humanos, precisamente como la plasmación formal fundamental de la idea de justicia, y a su pretendida universalidad, ante el dilema de reconocer la existencia de «derechos universales con uni-

\footnotetext{
${ }^{34}$ H. Albert, Tratado sobre la razón crítica, Buenos Aires, de. Sur, 1973, p. 27. J. Corominas, Etica primera, Bilbao, DDB, 2000, p. 87.

35 R. Rorty, Contingencia, ironía y solidaridad, trad. de A. Eduardo Sinnot y revisión de J. Vigil, Barcelona, Paidós, 1991.

${ }^{36}$ B. De Sousa Santos, De la mano de Alicia, op. cit., p. 106.

37 Sobre los distintos tipos de «relativismo», cfr. X. Etxeberría Mauleón, Ética de la diferencia, Bilbao, Universidad de Deusto, 1997, cap. V.
} 
formización cultural» $\mathrm{o}$ «diversidad cultural sin derechos universales» ${ }^{38}$. Algunos han tratado de ir «más allá» de ese posible debate, o de superarlo en aras de un cosmopolitismo fundamentado en la idea de incompletud ${ }^{39}$.

A este respecto, convendrá distinguir por un lado, en la formación del jurista, que una fundamentación relativa no implica ausencia de fundamentación, y en segundo lugar que la búsqueda permanente, en una carrera inalcanzable tras la justicia, es precisamente la expresión de un Derecho -y una justicia- que, en consonancia con la propia constitución de lo humano, no puede definirse sino como carencial, incompleta, inestable, o si se quiere, leve. ${ }^{40}$

\section{Rapidez}

Las transformaciones del discurso jurídico y la actividad de los juristas en la postmodernidad afectan también a la dimensión temporal de esa actividad, a la pluralidad de temporalidades de los sistemas jurídicos. En la actualidad, la hegemonía en esa pluralidad de tiempos jurídicos toma la forma de la precariedad, lo aleatorio, cuando no lo acelerado, la temporalidad característica de la reglamentación..$^{41}$ Como dice Boaventura de Sousa Santos, el siglo XX ha transformado el tiempo en falta de tiempo ${ }^{42}$. Es el «tiempo atemporal» de la sociedad red $^{43}$ : la experiencia de un Derecho nunca concluido y en permanente (casi inmediata) renovación -y en la que el jurista parece condenado a una labor sin fin como la de los condenados del

38 Vid. X. Etxeberria Mauleon, «El debate sobre la universalidad de los derechos humanos», en Varios, La Declaración Universal de Derechos Humanos en su cincuenta aniversario. Un estudio interdisciplinar, Bilbao, Universidad de Deusto, 1999, pp. 309-393. También J. González Amuchástegui, «¿Son los derechos humanos universales?», y A.-E. Pérez Luño, «Sobre la universalidad de los derechos humanos», ambos en Anuario de Filosofía del Derecho XV (1998), pp. 49-78 y 94-110 respectivamente.

39 B. De Sousa Santos, La globalización del Derecho, op. cit., p. 198-201.

${ }^{40}$ Es particularmente ilustrativo el trabajo de N. M ${ }^{\mathrm{a}}$ López Calera, «Naturaleza dialéctica de los derechos humanos», Anuario de Derechos Humanos 6 (1990), pp. 71-84.

${ }^{41}$ Vid. M. van de Kerchove y F. Ost, El sistema jurídico entre orden y desorden, trad. de I. Hoyo Sierra, Madrid, Universidad Complutense, 1988, pp. 189-194, que distinguen entre las múltiples temporalidades de los sistemas jurídicos y la relación entre la temporalidad jurídica y el nivel de organización de los sistemas. Por ejemplo, cabe distinguir entre el tiempo pasadista del juez, el tiempo futurista del legislador y el tiempo actual o presente de la Administración. Sobre el tiempo jurídico, véase además J. Ballesteros, Sobre el sentido del Derecho. Introducción a la Filosofía jurídica, Madrid, Tecnos, 1984, pp. 130-136; L. Carnelli, Tiempo y derecho, Buenos Aires, Valerio Abeledo editor, 1952, pp. 121-140.

42 B. De Sousa Santos, De la mano de Alicia, op. cit., p. 86.

43 «La sociedad red se caracteriza por la ruptura de la ritmicidad, tanto biológica como social, asociada con la noción de un ciclo vital». M. Castells, La era de la información, op. cit., pp. 463-503 (y especialmente pp. 498-503). 
Tártaro: Titón, Sísifo y Tántalo ${ }^{44}-$, la desaparición de la distancia temporal que conlleva la desaparición de la distancia espacial en la toma de decisiones «globales», la multiplicación de los asuntos que abarca el fenómeno jurídico y la multiplicación de las formas jurídicas a la hora de abordar esos asuntos, la inflación legislativa, la transmutación de la «atemporalidad» de la norma en contingencia, etc., todo ello parece abocar a un proceso de $d i$ solución de la unidad de tiempo en la actividad del jurista.

La prisa (o su total oposición: la parálisis) se convierte en la respuesta del jurista desconcertado o perplejo ante esa transformación del tiempo jurídico. El jurista se ve enfrentado así a una práctica jurídica cuya percepción temporal viene caracterizada por la urgencia y la permanente caducidad. A diferencia del derecho estable orientado al futuro -el derecho que permanece, que queda-, a diferencia del derecho «lento» que viene del pasado, de formación pausada y duradera, el derecho actual viene caracterizado por la mutabilidad, la rapidez, la caducidad que requiere un mundo en el que la urgencia es también lo característico en la economía, la ciencia, etc. (tal vez en consonancia con una cultura social en la que la lógica de la moda impone costumbres de usar y tirar, formas culturales pret-à-porter) ${ }^{45}$

A diferencia del jurista-artesano, que con todo el tiempo del mundo, busca afanosamente hasta encontrar la solución correcta para su caso, el jurista actual lucha a contratiempo por atrapar una solución. Y puesto que todo pasa rápidamente, se esfuerza en no perder el tren de un discurso y unas prácticas de cuya velocidad parece depender su éxito profesional. Esa percepción de lo temporal que caracteriza en general lo jurídico puede ser especialmente visible en el caso de la Universidad y los juristas-académicos, donde la carrera de fondo parece haber dejado paso a las pruebas de velocidad (o al salto de altura) ${ }^{46}$. Como si Sísifo, además de verse obligado a esa interminable condena de recomenzar siempre su tarea de hacer rodar la piedra con esfuerzo, tuviera que hacerlo -paradójicamente- a contrarreloj.

\section{Paradoja}

La multiplicidad, decíamos, conlleva la desaparición del punto de vista monista, del mito de la única solución, la disolución de las dicotomías clásicas en que se fundaba el discurso tradicional. Además, las formas rígidas, lineales, de lo jurídico, parecen dar paso a estrategias más sinuosas. Si uni-

\footnotetext{
${ }^{44}$ A.-J. Arnaud, «Los juristas frente a la sociedad (1975-1993)», op. cit., pp. 995-1000.

45 ¿Qué ocurre si no, por ejemplo, con la transformación de la noción de la costumbre jurídica, con la aparición de costumbres de rápida formación propias de la lex mercatoria?

${ }^{46}$ Sobre la prisa como característica de la actividad de los juristas, cfr. A. Nieto y T.-R. Fernández, El Derecho y el revés, op. cit., pp. 217-218.
} 
mos eso a la importancia que en los últimos años han cobrado las teorías que perciben el fenómeno jurídico en términos de circularidad ${ }^{47}$, no nos extrañaremos de esa perplejidad del jurista ante la constitución paradójica de un discurso que, en principio, estaba planteado como estrictamente racional; si acaso dialéctico.

Como dice Arnaud, si el Derecho es frágil, delicado, incierto, provisional, cambiante o inconstante, es a causa de sus paradojas internas, las paradojas que lo definen como tal derecho: entre lo universal y lo particular, lo individual y lo colectivo, la igualdad y la diferencia, o también entre lo nacional y lo local, la regulación y la desrregulación, lo formal y lo material... ${ }^{48}$, tal vez fruto de esa paradoja primera que es la de una técnica que pretende proteger la libertad limitándola; de modo que deshacer la paradoja es arriesgarse a deshacer el propio derecho. Para Arnaud, la paradoja de las paradojas tiene que ver con la para algunos incapacidad regulativa del derecho, su impotencia a la hora de abordar la complejidad: «El Derecho, tal como nosotros lo conocemos y lo practicamos, no está preparado para dirigir una sociedad moderna $»^{49}$. En todo caso, eso también supone la necesidad de introducir de algún modo en la formación del jurista una aproximación paradójica al fenómeno jurídico, al menos como un paso necesario para comprender un Derecho en el que la seguridad se da la mano con la incertidumbre. $^{50}$

De todos modos, conviene no dejarse seducir demasiado por los valores que apunta ese Derecho de la postmodernidad, que seguramente esconde cantos de sirena entre tanta polifonía ${ }^{51}$. Y si aludimos a ellos -a esos cinco

${ }^{47}$ La autorreferencialidad de Luhmann sería el ejemplo típico. Sobre la circularidad y autorreferencialidad del discurso jurídico, cfr. J. I. Martínez García, La imaginación jurídica, Madrid, Debate, 1992.

${ }^{48}$ A.-J. Arnaud, «Los juristas frente a la sociedad (1975-1993)», op. cit., pp. 1000-1004. Por ejemplo, véase una aproximación a los derechos fundamentales en términos de paradoja en R. de Asís Roig, Las paradojas de los derechos fundamentales como límites al poder, Madrid, Debate, 1992.

${ }^{49}$ A.-J. Arnaud, «Los juristas frente a la sociedad (1975-1993)», op. cit., p. 1003. ¿Alguna vez lo estuvo?, podríamos preguntarnos. Tal vez en una percepción así de lo jurídico subyace una imagen del Derecho que no se contrasta con la realidad histórica, como si a lo largo del tiempo el Derecho (tradicional) hubiera realmente dirigido las sociedades (tradicionales).

${ }^{50}$ Sobre la paradoja entre seguridad e incertidumbre legal cfr. L. Friedman, Total Justice, New York, Russell Sage, 1985; se hace recientemente eco de la misma J. J. Toharia, Opinión pública y Justicia, Madrid, CGPJ, 2001, pp. 25-26.

${ }^{51}$ Como dice Castells, «la flexibilidad puede ser una fuerza liberadora, pero también una tendencia represiva si quienes reescriben las leyes son siempre los mismos poderes»; y con pa- 
al menos, que sin duda podrían completarse o matizarse mucho más, y no es este el momento ${ }^{52}-$ es precisamente con esa intención. En ese sentido, creo que habría que dejar claras dos ideas de suma importancia. En primer lugar, estos cinco no son los únicos valores que definen hoy día el Derecho -y tal vez algunos dirán que no han sido bien escogidos-. El Código sigue vigente, podríamos decir, y esa especie de líquido glutinoso y futurista en que se ha convertido el discurso jurídico no ha llegado a todas partes (afortunadamente). Sin embargo, seguramente no nos equivocamos si decimos que esos valores (y algunos otros que los completan o desarrollan) tienden a hacerse hegemónicos en la forma y desarrollo del Derecho en la actualidad. Entre otras cosas -Pero Grullo obliga- porque son valores que tienden hegemónicamente a definir nuestra cultura en general, incluida en ella el sistema jurídico.

En segundo lugar, e insisto, si hacemos referencia a estas ideas no es porque deban ser asumidas en el sentido de «aceptadas» como tales, sino en el sentido de «hacerse cargo» de ellas. Es decir, aunque sea para oponerles resistencia ${ }^{53}$. Pero sabiendo que sólo se podrá transformar la realidad -si se desea- a partir de la propia realidad, y de las posibilidades inéditas de transformación que esta ofrece. Será mérito (y responsabilidad) de la enseñanza del derecho $-y$ de la enseñanza universitaria- estimular y desarrollar un espíritu crítico ante esa realidad, y reflexionar por tanto sobre los valores que deben orientar o impregnar la actividad jurídica (de ahí que no baste con una enseñanza que se reduzca a mera información o formación simplemente técnica). Y para ello, entre otras cosas, convendrá distinguir lo que de novedad o de ripio haya en muchas de las propuestas actuales, ya que esa inmediatez propia de nuestra cultura (y a la que antes nos referíamos) puede llevarnos a olvidar la génesis histórica de nuestros comportamientos y prácticas.

labras de Mulgan, «las redes se han creado no sólo para comunicar, sino también para ganar posición, para sobrecomunicar». M. Castells, La era de la información, op. cit., p. 89.

${ }^{52}$ Por ejemplo, tal vez podría añadirse la referencia a la virtualidad, forma de concebir en la actualidad la ficción constitutiva de lo jurídico (agradezco la sugerencia a la profesora $\mathrm{M}^{\mathrm{a}}$ José González Ordovás).

${ }^{53}$ Para una «política postmoderna de resistencia», vid. B. De Sousa Santos, De la mano de Alicia, op. cit., pp. 122 y sigs.

\section{DOXA 24 (2001)}

